\title{
The Influence of Emotional Inhibition on Intrusive Thoughts in a Non-Clinical Sample
}

\author{
Zahra Salehzadeh Einabad, ${ }^{1, *}$ and Mohammadreza Shairi ${ }^{1}$ \\ ${ }^{1}$ Clinical Psychology Department, Faculty of Humanities, Shahed University, Tehran, Iran \\ "Corresponding author: Zahra Salehzadeh Einabad, Clinical Psychology Department, Faculty of Humanities, Shahed University, Tehran, Iran. E-mail: z_salehzade@yahoo.com
} Received 2017 March 03; Revised 2017 April 23; Accepted 2017 July 09.

\begin{abstract}
Background: Given the studies insisting on the impacts of cultural issues on the emotion regulation strategies in eastern cultures and lack of study on the effects of emotional inhibition in our culture, this research aimed to investigate the influences of emotional inhibition on intrusive thoughts in non-clinical sample.

Methods: A quasi-experimental design was adopted with 45 participants that were randomly assigned to 2 groups (emotional inhibition and control groups). Participants which were selected according to cluster sampling answered to some questionnaires, including depression, general health, and emotion regulation. Then, a clip and a related instruction were presented for each group. They were asked to perform according to instruction after watching movie and tick on a paper whenever the thoughts of movie come to their mind. Data were analyzed using t test in SPSS-23.

Results: There are not significant differences between groups in terms of mood, emotion regulation, depression, and general health in the pre-test. Similarly, results indicated that there is not a significant differences between groups.

Conclusions: This research showed that the usefulness of emotional inhibition depends on the culture. In fact, in Eastern cultures, using emotion regulation strategies such as suppression and emotional inhibition are common so that avoidance is a short term and useful emotion regulation mechanism.
\end{abstract}

Keywords: Emotional Inhibition, Intrusive Thoughts, Emotion Regulation Strategies

\section{Background}

Despite the fact that people utilize a host of regulatory strategies, emotion inhibition has been the most typical focus of theory and research attention. Emotional inhibition is a generic strategy that tries to reduce reactions and does not require focus on a particular emotion or memory [1]. Emotion inhibition is considered "as the lack of emotional response to a stimulus that would typically evoke an emotional response" [2].

While, some researchers believe that the emotional inhibition is harmful, others assert that excessive emotion expressions may be disruptive. The psychological literature has been dominated by the assumption that the emotional inhibition leads to negative mental and physical health consequences [3]. Researchers [4] asserted that the emotional inhibition will lead to unpleasant health results. Noteworthy, the expression of emotion results in beneficial consequences, such as greater positive affect and improved immune functioning [4]. In emotional inhibition, not only is not adverse emotion expressed, but also it is sustained and is experienced as stress, leading to unfavorable health consequences. Prolonged inhibition of expression places a significant burden on the body and leads to vulnerability to stress-related diseases.
A salient unsolved issue is the effect of emotional inhibition on intrusive thoughts. While most studies have addressed to the impact of thought suppression, little is known about the the effect of emotional inhibition on cognitive functioning. On the other hand, Phung and Bryant [1] showed that emotion inhibition in comparison to control group did not lead to different amount of intrusive thought. A study [5] showed rumination and inhibitory dysfunctions are closely related. This research did not use experimental design and only investigated the relationship between rumination and inhibitory dysfunctions rather than emotional inhibition. Another study [6] reported Thought suppression immediately reduced thought frequency, but only in participants that had good inhibitory control. This study did not focus on the effects of emotional inhibition. Hence, effects of emotional inhibition have not been widely studied yet and most studies have not investigated the casual effects of emotional inhibition. On the other hand, researches about the effects of emotional inhibition are inconsistent $[3,4]$ and it is not clear whether psychological interventions can benefit from expression or inhibition of emotions.

It should be noted that cross-cultural studies have shown the importance of cultural differences in emotion 
regulation strategies such as emotional inhibition. For example, researchers [7] showed Japanese participants had fewer physiological symptoms when they were experiencing emotion. Similarly, researchers [8] demonstrated that Americans inhibited emotional expression to a greater extent than British participants. Also, a research [9] showed significant differences in either self-reported expressions or display rules in Black, Caucasian, Asian, and Hispanic individuals. Given the emphasis of cross-cultural studies on the role of culture in emotional regulation strategies $[7,9]$ and lack of research concerning the influence of emotional inhibition on the intrusive thoughts, it is necessary to investigate the effects of emotional inhibition on intrusive thoughts in Iranian culture. Therefore, the purpose of this study was to determine the effect of emotional inhibition on intrusive thoughts in non-clinical samples.

\section{Methods}

This study was conducted at Shahed University. Subjects were selected via cluster sampling from faculties of humanities, basic science and engineering. The informed consent was obtained from each subject. First, several classes were selected from classes of aforementioned faculties. Then, the sample was randomly selected from these classes and the subjects were randomly divided into two groups, with 21 individuals in the emotional inhibition group and 24 individuals in the control group. Subjects were assessed on depression, general health and emotional regulation inventories. Then, they were asked to evaluate their mood on a visual analogue scale and subsequently observed a clip. Then, they re-evaluated their mood on the visual analogue scale. Each subject received specific instructions according to literature $[1,10]$. They were asked to perform according to instruction after watching movie and tick on a paper whenever the thoughts of movie come to their mind. Then, reevaluation of mood and their adherence level to instruction were rated for each subject using visual analog scale [VAS]. Finally, subjects were presented with a gift as a mark of appreciation for participation in the study. Measures used in this study are as follows:

The Beck depression inventory-II [BDI-II]: Beck depression inventory-II [BDI-II] [11] is the revised version of Beck depression inventory developed to assess symptoms of depression. The coefficient alpha of the BDI-II was reported .91 [11]. This inventory also showed good validity. The researchers [12] reported that the BDI-II had positive correlations with self-report measures of depression and anxiety. Also, the researchers [13] obtained alpha coefficient of 0.93 and test-retest reliability of 0.93 in Iran. The validity of the inventory was confirmed. As a matter of fact, the correlation of this inventory was 0.873 with The Brief Symptom Inventory [BSI]. This questionnaire was used to ensure that there was no difference between groups in terms of this variable.

General health questionnaire [GHQ]: GHQ was developed in 1972 [14] to identify mental disorders in different centers and environments. A Cronbach's alpha of 76 was obtained, and validity of the test was confirmed [14]. The researchers [15] validated this test among university students in Iran. Cronbach's alpha of this questionnaire was 0.92. Similarly, convergent validity of the questionnaire was confirmed with general quality of life questionnaire $[\mathrm{r}$ $=-0.56]$. This questionnaire was used to ensure that there is no difference between groups in terms of this variable.

Emotion regulation questionnaire [ERQ]: ERQ [16] was developed to include strategies to evaluate style of response of individuals to stressful and adverse situations. The research identified two factors for style of response, reappraisal and suppression. The researchers [16] investigated psychometric features of this questionnaire in a sample of 416 Italian patients. Cronbach's alpha evaluation was 0.79 for reappraisal and 0.73 for suppression and the test-retest reliability for the whole scale was 0.69. A study in Iran [17] has confirmed validity and reliability of this questionnaire. The pattern of correlation coefficients between subscales and anxious thoughts indicated good concurrent validity of the Persian emotion regulation questionnaire. Factor analysis of this questionnaire showed two factors, including suppression and reappraisal which explained 49 per cent of the variance. Cronbach's alpha coefficients evaluations ranged from 0.60 to 0.81 . The questionnaire was used to ensure that there is no difference between groups in terms of this variable.

Visual analogue scale [VAS]: VAS was applied to determine the mood of subjects and their adherence on this continuum. In one study [1], subjects were asked to determine their mood on the following scale, 0 : without any distress, 100: extremely distressed]. Subjects also determined level of adherence to instructions [0: no adherence to, 100: full adherence].

A video clip of a grizzly bear attack [from "Faces of Death"]: A video clip about a bear attacking a tourist was taken from a documentary named "Faces of Death". This documentary presents videos from real life and traumatic events which were recorded incidentally. This clip shows a bear attacking a tourist and ends with vague scenes showing the attack. The pilot study proved that the clip is emotionally charged and causes negative feelings [18]. The film has been studied in Iranian culture [19]. This film was dubbed and prepared by Transe24 Company. Women considered the film to be sadder, more despicable, more 
unpleasant, more negative, more nerve shattering and anxiety-provoking.

First, descriptive statistics, such as mean and standard deviation were analyzed in SPSS-23. Then, in order to compare two groups t test was conducted.

\section{Results}

Table 1 contains sample size, gender, age of sample, and distribution of individuals in each of the two groups. The observed $t$ for age was 1.35 , which is not significant compared with critical values.

Descriptive findings related to questionnaires and mood of subjects are presented in Table 2 . These variables were measured before providing an independent variable.

In addition, descriptive findings related to intrusive thoughts are presented in Table 2.

The results in Table 3 show no significant difference between groups in variables, including mood, adherence, depression, emotional regulation, and mental health. Results of t test did not show a significant difference in terms of intrusive thoughts.

\section{Discussion}

This study showed that intrusive thoughts have not been different in 2 groups. Emotion inhibition in comparison to control group did not lead to a significant decrease or increase of intrusive thoughts. This result is consistent with some researches $[1,20,21]$ - the first study was conducted in China- and inconsistent with a research [22]-that focused on clinical sample. A researcher [20] investigated if deficits in the inhibition of irrelevant emotional stimuli in working memory are related to individual differences in ruminative responses to negative mood states. Response style questionnaire [RSQ] was used in this study. Results showed that only were significant inhibition in the participants who scored low on the RSQ. These group differences were significant after controlling for current depression scores. This result suggests that rumination and inhibitory dysfunctions are closely related. Another study [21] investigated if suppression of disgust related thoughts, in comparison with no suppression, influenced thought frequency and emotional responses, and whether this result was correlated with participants' cognitive inhibition abilities. They reported that thought suppression immediately reduced thought frequency, but only in participants that had good inhibitory control. People with higher levels of inhibition are likely to perceive the outside world as threatening, because they predict unsatisfactory reactions from others. So, they attempt to avoid these experiences and reactions and choose self-enhancing strategies like withdrawal. Researchers suggested that avoidance may have a negative effect. They considered avoidance as the core of most psychological disorders. Avoidance and inhibition is a maladaptive response in the face with stressors. It is also a risk factor for distress [i.e. anxiety and depression] and maladaptive behaviors. A study [22] examined the effectiveness of acceptance and commitment therapy in obsessive-compulsive disorder and showed the significant decreases in obsessive compulsive scale, depression and anxiety inventories in post test. Process of treatment and results from this study suggest that acceptance and commitment therapy can be an effective intervention for difficult thoughts, feelings, and behaviors seen in obsessivecompulsive disorder.

In order to explain the findings, it should be noted that cultural issues may explain the results. Cross-cultural literature shows that emotion regulation strategies are highly affected by collectivism and individualism [23, 24]. In fact, Eastern participants in comparison to European and American participants are more inclined to use suppression. It has been noted that not only is suppression widespread among Asian participants, but also they use suppression as a strategy to regulate negative emotions [25]. Some studies indicate that Asian participants often express lower emotional reactions to emotion provoking tasks in comparison to other cultural groups. In fact, it is possible that participants in control group use the same strategy as the emotional inhibition group.

Secondly, this result is consistent with the hypothesis that avoidance is a short term and useful emotion regulation mechanism [26, 27]. As we mentioned earlier, there are inconsistent result concerning usefulness of emotional inhibition. While some researchers insisted that emotional inhibition has detrimental effects for mind and body, this research showed that the usefulness of emotional inhibition depends on the culture. In our study and another study [1] which has been carried out in china it has been proved that emotional inhibition does not have any influence on thought suppression.

This study showed that there was no significant difference between emotional inhibition and control group. This finding is inconsistent with studies that show negative consequences of emotional inhibition. For example, negative emotional inhibition is correlated with increased risk of several diseases [28]. On the other hand, expressing anger is correlated with increased risk of cardiovascular disease [29]. Similarly, people that express anger may suffer from negative health consequences [30]. Researchers [31] suggested the way that anger is related to coronary heart disease, hypertension, and cancer. A study [32] revealed that there is a positive correlation between cardio- 
Table 1. Descriptive Findings Relating to the Frequency of Subjects in Each Group, Gender, and Age of Subjects ${ }^{\mathrm{a}}$

\begin{tabular}{|c|c|c|c|c|}
\hline \multirow[t]{2}{*}{ Group } & \multirow[t]{2}{*}{ Sample Size } & \multicolumn{2}{|c|}{ Gender } & \multirow[t]{2}{*}{ Age } \\
\hline & & Man & Woman & \\
\hline Control & 24 & $12(50)$ & $12(50)$ & $20.41 \pm 1.08$ \\
\hline Emotional inhibition & 21 & $10(47.6)$ & $11(52.4)$ & $19.57 \pm 1.24$ \\
\hline
\end{tabular}

${ }^{\mathrm{a}}$ Values are expressed as No. (\%) or mean $\pm \mathrm{SD}$.

Table 2. Findings Relating to Research Variables ${ }^{\mathrm{a}}$

\begin{tabular}{lcc}
\hline Variable & Control & Emotional Inhibition \\
\hline BDI-II & $11.02 \pm 8.47$ & $10.57 \pm 7.23$ \\
\hline ERQ-suppression & $12.83 \pm 5.58$ & $13.61 \pm 4.88$ \\
\hline GHQ-12 & $10.54 \pm 4.79$ & $11.80 \pm 5.28$ \\
\hline Adherence & $73.54 \pm 20.56$ & $76.15 \pm 15.32$ \\
\hline Mood & $38.62 \pm 21.20$ & $34.25 \pm 23.01$ \\
\hline Intrusive thoughts & $37.95 \pm 39.15$ & $26.09 \pm 21.76$ \\
\hline
\end{tabular}

Abbreviations: BDI-II, Beck depression inventory-II, ERQ, emotion regulation questionnaire; GHQ, general health questionnaire.

${ }^{\mathrm{a}}$ Values are expressed as mean $\pm \mathrm{SD}$.

vascular disease and expression of anger. It should be noted that the way how emotions are experienced and expressed during psychotherapeutic sessions is of great importance and expression in an unstructured fashion can be deleterious [33]. Research about crying do not show emotional expression is beneficial for health [34]. Researchers [35] showed that suppression of specific emotional experiences has a mediating role in the relationship between well-being and emotional suppression tendency. In addition, suppression tendency explained $30 \%$ of the individual differences in emotional suppression of specific experiences. This result suggests that other factors, such as personality traits are likely to affect emotional suppression. It should be noted that interpretation of the emotional inhibition-health link is very complicated. In fact, there are inconsistent findings in this field. It can be possible that there are some mediators in this link such as gender, personality traits and cultural differences.

Noteworthy, there are some limitations in this study. First, the research sample in each group was limited. Especially both genders participated in the research. Secondly, the studied sample consisted of non-clinical students with specific education and specific demographic characteristics and so results may not be applicable to generalization. Thirdly, using specific tools and showing the clip of a bear attacking a tourist can be considered as a limitation. And finally, the length of instruction given each group was un- equal.

The following suggestions are presented: first, a larger sample with educational, occupational, age and gender diversity for higher generalization is recommended. Secondly, a research on various clinical samples according to the literature is recommended. Thirdly, different visual and verbal stimuli should be primed to create emotional conditions.

\section{Acknowledgments}

All procedures performed in this study involving human participants were in accordance with the ethical standards of the institutional and /or national research committee and with the 1964 Helsinki declaration and its later amendments or comparable ethical standards. This research was confirmed in a session on 14/12/2015 with the number of 207 in faculty of humanitarian sciences at Shahed University.

\section{Footnotes}

Authors' Contribution: All authors had equal role in design, work, statistical analysis and manuscript writing. Conflict of Interest: The authors declare no conflict of interest.

Funding/Support: Shahed University. 
Table 3. T Test

\begin{tabular}{|c|c|c|c|c|c|c|c|c|c|}
\hline \multirow[t]{3}{*}{ Variable } & \multicolumn{2}{|c|}{ Levene's Test $^{a}$} & \multicolumn{7}{|c|}{ T-Test $^{\mathbf{b}}$} \\
\hline & \multirow[t]{2}{*}{$\mathbf{F}$} & \multirow[t]{2}{*}{ Sig. } & \multirow[t]{2}{*}{ t } & \multirow[t]{2}{*}{ df } & \multirow[t]{2}{*}{ Sig. } & \multirow[t]{2}{*}{ Mean Difference } & \multirow[t]{2}{*}{ Std. Error Difference } & \multicolumn{2}{|c|}{$95 \% \mathrm{CI}^{\mathrm{C}}$} \\
\hline & & & & & & & & Lower & Upper \\
\hline BDI-II & 0.278 & 0.600 & 0.190 & 43 & 0.851 & 0.44882 & 2.36819 & -4.32 & 5.224 \\
\hline ERQ-suppression & 0.713 & 0.403 & -0.500 & 43 & 0.620 & -0.78763 & 1.57474 & -3.963 & 2.388 \\
\hline GHQ-12 & 0.007 & 0.936 & -0.844 & 43 & 0.403 & -1.26786 & 1.50249 & -4.297 & 1.762 \\
\hline Adherence & 1.49 & 0.229 & -0.484 & 43 & 0.631 & -2.64881 & 5.47176 & -13.68 & 8.38 \\
\hline Mood & 0.207 & 0.651 & 0.803 & 43 & 0.426 & 5.94643 & 7.40117 & -8.979 & 20.872 \\
\hline Intrusive thoughts & 2.63 & 0.112 & 1.231 & 43 & 0.225 & 11.86310 & 9.63783 & -7.573 & 31.299 \\
\hline
\end{tabular}

\section{References}

1. Phung SQ, Bryant RA. The influence of cognitive and emotional suppression on overgeneral autobiographical memory retrieval. Conscious Cogn. 2013;22(3):965-74. doi: 10.1016/j.concog.2013.06.008. [PubMed: 23871861].

2. Bryant RA, Kapur A. Hypnotically induced emotional numbing the roles of hypnosis and hypnotizability. Int J Clin Exp Hypn. 2006;54(3):281-91. doi: 10.1080/00207140600689462. [PubMed 16858904].

3. Greenberg MA, Stone AA. Emotional disclosure about traumas and its relation to health: effects of previous disclosure and trauma severity. J Pers Soc Psychol. 1992;63(1):75-84. [PubMed: 1494986].

4. Pennebaker JW, Seagal JD. Forming a story: the health benefits of narrative. J Clin Psychol. 1999;55(10):1243-54. doi: 10.1002/(SICI)10974679(199910)55:10<1243::AID-JCLP6>3.0.CO;2-N. [PubMed: 11045774].

5. Haynes SG, Feinleib M, Kannel WB. The relationship of psychosocial factors to coronary heart disease in the Framingham Study. III. Eight-year incidence of coronary heart disease. Am J Epidemiol 1980;111(1):37-58. [PubMed: 7352459].

6. Jorgensen RS, Johnson BT, Kolodziej ME, Schreer GE. Elevated blood pressure and personality: a meta-analytic review. Psychol Bull. 1996;120(2):293-320. [PubMed: 8831299].

7. Pennebaker JW, Traue HC. In: Emotion, Inhibition, and Health. Traue HC, Pennebaker JW, editors. Seattle: Hogrefe and Huber Publishers; 1993. Inhibition and psychosomatic processes.

8. Carver CS, Scheier MF, Weintraub JK. Assessing coping strategies: a theoretically based approach. J Pers Soc Psychol. 1989;56(2):267-83. [PubMed: 2926629].

9. Wenzlaff RM, Wegner DM. Thought suppression. Annu Rev Psychol. 2000;51:59-91. doi: 10.1146/annurev.psych.51.1.59. [PubMed:10751965]

10. Dunn BD, Billotti D, Murphy V, Dalgleish T. The consequences of effortful emotion regulation when processing distressing material: a comparison of suppression and acceptance. Behav Res Ther. 2009;47(9):761-73. doi: 10.1016/j.brat.2009.05.007. [PubMed 19559401].

11. Beck AT, Steer RA, Ball R, Ranieri W. Comparison of Beck Depression Inventories -IA and -II in psychiatric outpatients. J Pers Assess. 1996;67(3):588-97. doi: 10.1207/s15327752jpa6703_13. [PubMed 8991972].

12. Storch EA, Roberti JW, Roth DA. Factor structure, concurrent validity, and internal consistency of the beck depression inventory?second edition in a sample of college students. Depression Anxiety. 2004;19(3):187-9. doi:10.1002/da.20002.

13. Dobson KS, Mohammadkhani P. Psychometric properties of the BDIII in a sample of patients with major depression disorder. J Rehabil. 2007;8(2):80-6.
14. Sánchez-Lopez M, Dresch V.The12-Item General Health Questionnaire (GHQ-12): reliability, external validity and factor structure in the Spanish population. Psicothema. 2008;20(4).

15. Yaghubi H, Karimi M, Omidi A, Barooti E, Abedi M. Validity and factor structure of the General Health Questionnaire (GHQ-12) in university students. Int J Behav Sci. 2012;6(2):153-60.

16. Balzarotti S, John OP, Gross JJ. An Italian Adaptation of the Emotion Regulation Questionnaire. Eur J Psychol Assess. 2010;26(1):61-7. doi: 10.1027/1015-5759/a000009.

17. Ghasempour A, Ilbeigi R, Hassanzadeh S. The psychometric properties of Gross and John Emotion Regulation Questionnaire in an Iranian sample. The sixth congress of Mental Health. University of Gilan; 2011. pp. 724-2.

18. Gratz KL, Rosenthal MZ, Tull MT, Lejuez CW, Gunderson JG. An experimental investigation of emotion dysregulation in borderline personality disorder. J Abnorm Psychol. 2006;115(4):850-5. doi: 10.1037/0021843X.115.4.850. [PubMed: 17100543].

19. Salehzadeh Einabad Z, Shairi M. The processing of a emotional clip in a nonclinical sample. [in Persian]. Journal of Rooyesh-e- Ravanshenasi. ;In press(In press).

20. Joormann J. Differential Effects of Rumination and Dysphoria on the Inhibition of Irrelevant Emotional Material: Evidence from a Negative Priming Task. Cogn Ther Res. 2006;30(2):149-60. doi: 10.1007/s10608-006-9035-8.

21. Olafsson RP, Emmelkamp PM, Gunnarsdottir ER, Snaebjornsson T, Olason DT, Kristjansson A. Suppressing disgust related thoughts and performance on a subsequent behavioural avoidance task: implications for OCD. Behav Res Ther. 2013;51(3):152-60. doi: 10.1016/j.brat.2012.11.008. [PubMed: 23337184].

22. Izadi R, Asgari K, Neshatdust H, Abedi M. The Effect of acceptance and commitment therapy on the frequency and severity of symptoms of obsessive compulsive disorder. Zahedan J Res Med Sci. 2012;14(10):10712.

23. Matsumoto D. Ethnic differences in affect intensity, emotion judgments, display rule attitudes, and self-reported emotional expression in an American sample. Motiv Emot. 1993;17(2):107-23. doi: 10.1007/bfo0995188.

24. Kitayama S, Markus HR, Kurokawa M. Culture, Emotion, and Wellbeing: Good Feelings in Japan and the United States. Cogn Emot. 2000;14(1):93-124. doi:10.1080/026999300379003.

25. Butler EA, Lee TL, Gross JJ. Emotion regulation and culture: are the social consequences of emotion suppression culture-specific? Emotion. 2007;7(1):30-48. doi: 10.1037/1528-3542.7.1.30. [PubMed: 17352561].

26. Williams JM, Barnhofer T, Crane C, Herman D, Raes F, Watkins E, et al. Autobiographical memory specificity and emotional disorder. Psychol Bull. 2007;133(1):122-48. doi:10.1037/0033-2909.133.1.122. [PubMed: 17201573]. 
27. Williams JM, Teasdale JD, Segal ZV, Soulsby J. Mindfulness-based cognitive therapy reduces overgeneral autobiographical memory in formerly depressed patients. J Abnorm Psychol. 2000;109(1):150-5. [PubMed: 10740947].

28. Berry DS, Pennebaker JW. Nonverbal and verbal emotional expression and health. Psychother Psychosom. 1993;59(1):11-9. [PubMed: 8441791].

29. Siegman AW, Anderson RA, Berger T. The angry voice: its effects on the experience of anger and cardiovascular reactivity. Psychosom Med. 1990;52(6):631-43. [PubMed: 2287702].

30. Schwartz GE, Weinberger DA, Singer JA. Cardiovascular differentiation of happiness, sadness, anger, and fear following imagery and exercise. Psychosom Med. 1981;43(4):343-64. [PubMed: 7280162].

31. Thomas SP, Williams RL. Perceived stress, trait anger, modes of anger expression, and health status of college men and women. Nurs Res. 1991;40(5):303-7. [PubMed: 1896331]
32. Gidron Y, Davidson K, Ilia R. Development and cross-cultural and clinical validation of a brief comprehensive scale for assessing hostility in medical settings. J Behav Med. 2001;24(1):1-15. [PubMed: 11296467].

33. Littrell J. Is the reexperience of painful emotion therapeutic? Clin Psychol Rev. 1998;18(1):71-102. [PubMed: 9455624].

34. Poles MA, Elliott J, Vingerhoets J, Michiels L, Scholliers A, Bloor S, et al. Despite high concordance, distinct mutational and phenotypic drug resistance profiles in human immunodeficiency virus type 1 RNA are observed in gastrointestinal mucosal biopsy specimens and peripheral blood mononuclear cells compared with plasma. J Infect Dis. 2001;183(1):143-8. doi: 10.1086/317640. [PubMed: 11087199].

35. Stupar S, van de Vijver FJR, Fontaine JRJ. Emotional suppression and well-being in immigrants and majority group members in the Netherlands. Int J Psychol. 2014;49(6):503-7. 